\title{
APLICACIÓN DE LA BÚSQUEDA TABÚ EN LA RESOLUCIÓN DEL PROBLEMA CAPACITADO DE LOTIFICACIÓN EN SISTEMAS DE PRODUCCIÓN MULTINIVEL: UN ESTADO DEL ARTE
}

\author{
DOI: http://doi.org/10.17981/bilo.2.1.2020.1
}

Fecha de Recepción: 01/12/2019. Fecha de Aceptación: 15/01/2020. Fecha de Publicación:17/01/2020

\author{
Alfonso Romero-Conrado ${ }^{1}$ (iD) https://orcid.org/0000-0003-4603-0785, Jairo R. Coronado-Hernández ${ }^{1}$ \\ https://orcid.org/0000-0003-4360-6128 y Renso Raúl Visbal-Acevedo ${ }^{2}$ (iD) https://orcid.org/0000-0002-1753-5103 \\ ${ }^{1}$ Universidad de la Costa CUC, Departamento de Gestión Industrial, Agroindustrial y Operaciones. Barranquilla \\ Universitat Politècnica de València. (España) \\ , (Colombia) aromero17@ cuc.edu.co, jcoronad18@cuc.edu.co \\ ${ }^{2}$ Universidad del Valle, Cali, (Colombia) renso.visbal@ correounivalle.edu.co
}

Para citar este artículo:

A. Romero-Conrado, J. Coronado-Hernández , R. Visbal-Acevedo "Aplicación de la búsqueda tabú en la resolución del problema capacitado de lotificación en sistemas de producción multinivel: Un estado del arte", BILO, vol.

2, no. 1, 2020. DOI: http://doi.org/10.17981/bilo.2.1.2020.1

\section{Resumen}

En este trabajo, se aborda desde la literatura, un estudio de la aplicación de la búsqueda tabú para la resolución del proble ma de planificación de la producción en sistemas multinivel con restricciones de capacidad. Esta familia de problemas está clasificada dentro de la literatura como Np-Hard. Las estrategias de relajación y búsqueda, especialmente la Búsqueda tabú, han jugado un papel determinante en el desarrollo de alternativas de solución de este tipo de problemas, permitiendo obtener de soluciones cercanas al valor óptimo de mínimo costo, en tiempos operativamente aceptables. El presente artículo tiene por objetivo recopilar, describir y analizar el uso y aplicación de la búsqueda tabú en la solución del problema de lotificación en sistemas de producción multinivel. Contemplando la gran cantidad de variaciones del problema y haciendo énfasis en la metodología utilizada y la configuración de la búsqueda, se pretende establecer la línea base para el desarrollo de nuevos algoritmos de solución para el problema delotificación.

\section{Palabras clave}

Búsqueda Tabú; Lotificación; Sistema Multinivel; Restricciones de Capacidad.

\begin{abstract}
In this work, a study of the application of the taboo search for the resolution of the problem of production planning in multilevel systems with capacity restrictions is approached from the literature. This family of problems is classified in the literature as Np-Hard. Relaxation and search strategies, especially Taboo Search, have played a decisive role in the development of alternative solutions for this type of problem, allowing to obtain solutions close to the optimal minimum cost value, in operationally acceptable times. The purpose of this article is to collect, describe and analyze the use and application of taboo search in the solution of the problem of lotification in multilevel production systems. Contemplating the large number of variations of the problem and emphasizing the methodology us ed and the configuration of the search, it is intended to establish the baseline for the development of new solution algorithms for the lotification problem.
\end{abstract}

\section{Key Words}

Taboo Search; Lotification; Multilevel System; Capacity Restrictions. 


\section{INTRODUCCIÓN}

La solución al problema de lotificación es uno de temas más estudiados dentro de la literatura científica referente a la planificación de la producción. La complejidad de este tipo de problemas está determinada principalmente por el tamaño del problema, la cantidad de productos y su estructura, el comportamiento de su demanda y las diversas restricciones del proceso. El uso de meta heurísticas permite la obtención de soluciones cercanas a valores óptimos en tiempos relativamente cortos, resultando factibles para intervalos de planeación operativa. La búsqueda Tabú es una de las meta heurísticas más utilizadas en la solución de problemas de planificación de la producción y dadas sus ventajas, su aplicación es frecuentemente recomendada como trabajo futuro de investigación.

El presente artículo tiene por objetivo recopilar, describir y analizar algunas de las aplicaciones de la búsqueda tabú presentes en la literatura con el fin de caracterizar su eficiencia y funcionamiento en la solución de problemas de lotificación. Mediante un análisis de las metodologías utilizadas y la configuración de los parámetros básicos de la búsqueda tabú se pretende establecer una línea base para el desarrollo de nuevos algoritmos de solución para el problema de lotificación en sistemas de producción multinivel.

En la sección 2 se mostrará una breve descripción de la metodología de búsqueda utilizada. En la sección 3 se describirán algunas de las aplicaciones de la búsqueda tabú en problemas de lotificación. En la sección 4 se mostrará un resumen de los resultados y se analizarán las tendencias metodológicas de los aportes estudiados. En la sección 5 se expondrán las conclusiones y las sugerencias para trabajos futuros.

\section{METODOLOGÍA}

\section{A. Metodología de búsqueda}

La consulta a fuentes bibliográficas primarias y secundarias se realizó a través de las bases de datos especializadas de Science Direct y Scopus. En la búsqueda se utilizaron términos como: ("Multilevel” OR "Multi-level”) AND ("Lotsizing” OR "Lotsizing”); ("Lot-sizing” OR “Lotsizing”) AND (“Tabu”).

De los resultados de la búsqueda, se seleccionaron aquellos artículos de investigación y revisiones de literatura que exponían la aplicación de la búsqueda tabú en problemas de lotificación o presentaban una lista de referencias relacionadas.

\section{APLICACIÓN DE LA BÚSQUEDA TABÚ EN LA SOLUCIÓN DEL PROBLEMA DE LOTIFICACIÓN.}

Desde sus orígenes a finales de la década de los ochenta y finales de los noventa [1]-[3], la búsqueda tabú ha sido ampliamente utilizada en la búsqueda de mejores soluciones para problemas de lotificación de gran complejidad. A continuación, se mencionan y describen algunos de los aportes más relevantes en modelos de lotificación.

\section{A. Sistemas de producción de un solo nivel}

Hindi [4] presentó la solución de un problema de lotificación capacitado de un solo ítem con demanda dinámica, considerando costos fijos de inicio y utilización. En su aporte, se aplicó un algoritmo simple de búsqueda tabú, que partía de una solución inicial factible obtenida mediante un modelo de transbordo. Los resultados fueron comparados con los arrojados mediante un algoritmo simplex dual, demostrando una mayor eficiencia computacional en el uso de la búsqueda tabú.

Más adelante, este mismo autor [5] aplicó el procedimiento de búsqueda tabú en la solución de un problema capacitado con demanda dinámica y múltiples ítems (CLSP). Se modeló el problema mediante programación entera mixta MIP y se aplicó un método de relajación mediante generación de columnas. Esta solución fue el punto de partida para el algoritmo de búsqueda tabú, el cual utiliza una lista tabú del tamaño del número de periodos planificados, criterios de aspiración por objetivo y un criterio de parada determinado por un número de iteraciones sin mejoras sustanciales en la función objetivo.

El aporte [6] muestra la aplicación de la búsqueda tabú en un problema de lotificación multi ítem con tiempos de alistamiento (Setup Carry-over). La búsqueda tabú consistió en cinco movimientos básicos, para secuenciación y la definición del tamaño del lote. A su vez se utilizaron listas tabú dinámicas, memoria adaptativa y penalidades autoajustadas para fortalecer la búsqueda.

En [7] se utiliza la búsqueda tabú para la solución de problemas de planificación de la producción con costos de alistamiento y múltiples ítems. Basados en la solución del problema mediante método simplex, los autores proponen un 
BILO Vol. 2 no. 1, Enero- Junio de 2020

método mejorado para la determinación de listas de candidatos para la exploración de vecindarios de solución y la obtención de soluciones con menor gasto computacional.

Karimi et al. [8] exponen la utilización de la búsqueda tabú para la solución del problema identificado por ellos como CLSP+: un problema de lotificación multi-ítem, con un solo nivel, restricciones de capacidad y demanda dinámica, que tenía como consideración especial la presencia de pedidos atrasados (Backlogging) y similitudes en los requerimientos de alistamiento (Setup Carry-Over). En este caso, el problema es abordado de manera similar a [5], y está formulado como un modelo de programación entera mixta MIP y se obtienen soluciones iniciales factibles por medio de algoritmos heurísticos que tienen en cuenta los cambios en la demanda, las reglas de lotificación, las condiciones de factibilidad y la determinación de los ítems con Setup Carry Over. Luego la solución inicial es mejorada aplicando un modelo de minimum-cost network flow. Esta solución es utilizada como inicio del procedimiento de búsqueda tabú, configurado con el número de movimientos arrojados por el modelo de mínimo costo, logrando tiempo soluciones cercanas a valores óptimos (gaps cercanos al 1,3\% para ciertos casos).

En la referencia [9] se presenta un algoritmo de búsqueda tabú para el problema dinámico de lotificación con devoluciones de producto y re-manufactura (DLRR). Utilizando un algoritmo de cadena de bloques (block-chains) se generan las soluciones iniciales para el procedimiento de búsqueda tabú. El algoritmo arrojó resultados óptimos en el $96 \%$ de los problemas de prueba y una baja desviación estándar comparado con soluciones previas del problema estudiado.

\section{B. Sistemas de producción Multinivel}

Uno de los primeros aportes registrados fue el de [10], este presenta la aplicación del recocido simulado y la búsqueda tabú en la solución del problema de lotificación multinivel para sistemas de ensamble con cuello de botella. El problema es modelado inicialmente mediante programación lineal LP y se utiliza un algoritmo voraz modificado en conjunto con ambas meta heurísticas para la obtención de soluciones iniciales. Los resultados de las meta heurísticas demostraron ser superiores en eficiencia con respecto al uso de LP.

Kimms [11] propuso dos meta heurísticas para la solución del problema de lotificación y scheduling en sistemas multinivel con una sola máquina, múltiples ítems y restricciones de capacidad: la primera está basada en la heurística de "randomized regrets" y la segunda se basa en un procedimiento de búsqueda tabú. Para esta última se tiene en cuenta una lista de materiales representada mediante un diagrama de Gozinto, de la cual se define una estructura de arcos disyuntivos para dar solución al problema. Los resultados entre las dos meta heurísticas propuestas fueron muy similares entre sí y cercanos a los valores óptimos.

En la referencia [12] se aplica la búsqueda tabú, el recocido simulado y algoritmos genéticos al problema de lotificación multinivel con varias clases de demanda. Los resultados obtenidos permitieron afirmar que los algoritmos de búsqueda tabú tuvieron un mejor desempeño en problemas con demanda conformada por órdenes confirmadas.

En el aporte [13]-[16], se muestra el uso de meta heurísticas para el problema de lotificación multinivel con restricciones de capacidad, con estructuras generales de productos, tiempos y costos de alistamiento y tiempos de entrega. Se utilizaron la búsqueda tabú y el recocido simulado para la solución del problema y los resultados fueron comparados posteriormente con los obtenidos mediante heurísticas previamente utilizadas para la solución del problema. Las meta heurísticas arrojaron soluciones muy cercanas al óptimo para problemas pequeños.

\section{RESULTADOS}

Las tablas I y II resumen la configuración básica de la búsqueda tabú utilizada en los artículos consultados, para sistemas de producción de un solo nivel y de múltiples niveles respectivamente.

\begin{tabular}{|c|c|c|c|c|c|c|}
\hline Referencia & $\begin{array}{l}\text { Solución de } \\
\text { Referencia }\end{array}$ & $\begin{array}{l}\text { Solución } \\
\text { Inicial }\end{array}$ & Movimientos & $\begin{array}{l}\text { Métodos de } \\
\text { Intensificación }\end{array}$ & $\begin{array}{l}\text { Métodos de } \\
\text { Diversificación }\end{array}$ & $\begin{array}{l}\text { Método de } \\
\text { Aspiración }\end{array}$ \\
\hline [4] & $\begin{array}{c}\text { Algoritmo } \\
\text { Simplex Dual }\end{array}$ & $\begin{array}{c}\text { Solución a } \\
\text { problema de } \\
\text { transbordo }\end{array}$ & $\begin{array}{l}\text { Vecinos con } \\
\text { mejor solución } \\
\text { a problema de } \\
\text { transbordo }\end{array}$ & $\begin{array}{l}\text { Mostraron no } \\
\text { ser necesarios }\end{array}$ & $\begin{array}{l}\text { Mostraron no } \\
\text { ser necesarios }\end{array}$ & $\begin{array}{l}\text { Aspiración } \\
\text { por mejora } \\
\text { en función } \\
\text { objetivo }\end{array}$ \\
\hline [5] & $\begin{array}{l}\text { Programación } \\
\text { entera mixta }\end{array}$ & $\begin{array}{l}\text { Método de } \\
\text { Generación } \\
\text { de Columnas }\end{array}$ & $\begin{array}{l}\text { Lista de } \\
\text { candidatos con } \\
\text { menores } \\
\text { costos de } \\
\text { setup }\end{array}$ & $\begin{array}{c}\text { No se } \\
\text { especifica }\end{array}$ & $\begin{array}{l}\text { Problema de } \\
\text { transbordo sin } \\
\text { restricciones } \\
\text { de capacidad }\end{array}$ & $\begin{array}{l}\text { Aspiración } \\
\text { por mejora } \\
\text { en función } \\
\text { objetivo }\end{array}$ \\
\hline
\end{tabular}


BILO Vol. 2 no. 1, Enero- Junio de 2020

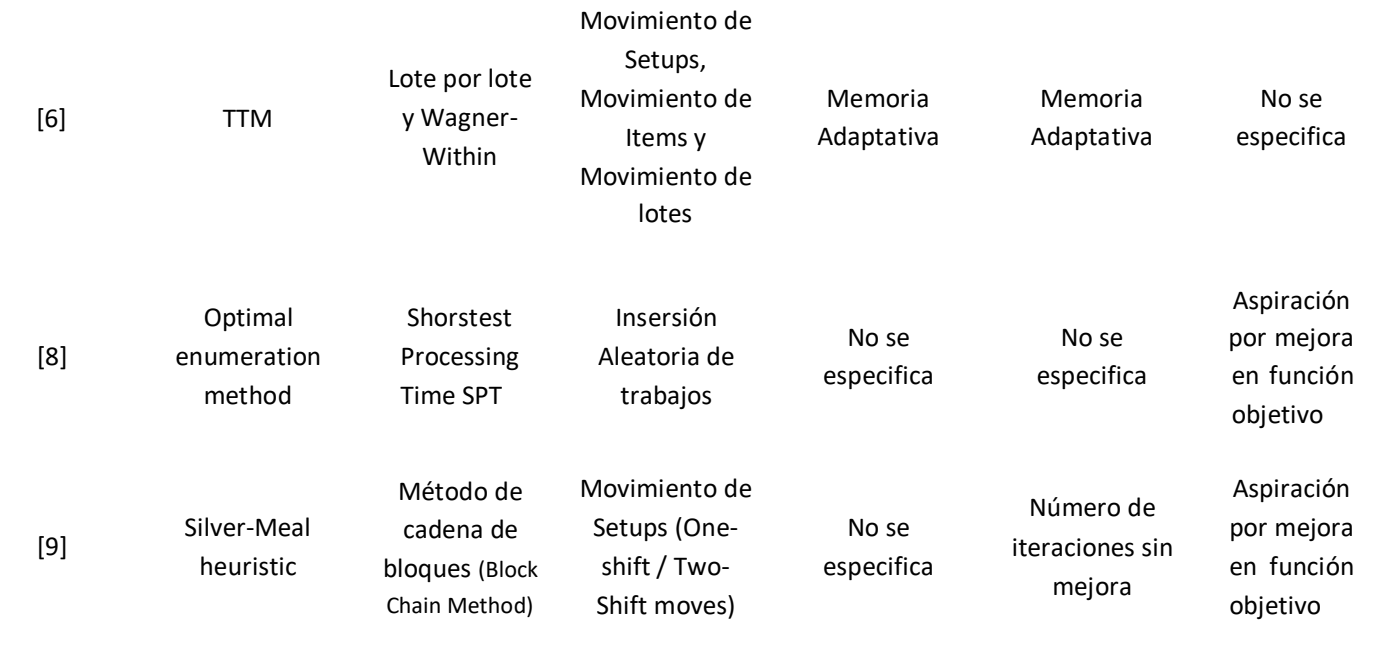

Tabla 1. Configuraciones básicas de la búsqueda tabú en modelo de lotificación de un solo nivel. Fuente: Autores

\begin{tabular}{|c|c|c|c|c|c|c|}
\hline Referencia & $\begin{array}{l}\text { Solución de } \\
\text { Referencia }\end{array}$ & $\begin{array}{l}\text { Solución } \\
\text { Inicial }\end{array}$ & $\begin{array}{c}\text { Movimientos } \\
\text { en la } \\
\text { búsqueda }\end{array}$ & $\begin{array}{c}\text { Método de } \\
\text { Intensificación }\end{array}$ & $\begin{array}{l}\text { Método de } \\
\text { Diversificación }\end{array}$ & $\begin{array}{l}\text { Método de } \\
\text { Aspiración }\end{array}$ \\
\hline [10] & $\begin{array}{l}\text { Programación } \\
\text { Lineal LP }\end{array}$ & $\begin{array}{l}\text { Algortimo } \\
\text { Voraz } \\
\text { modificado }\end{array}$ & $\begin{array}{l}\text { Movimiento } \\
\text { No tabú con } \\
\text { mayor valor } \\
\text { en Función } \\
\text { Objetivo }\end{array}$ & No se especifica & $\begin{array}{c}\text { No se } \\
\text { especifica }\end{array}$ & $\begin{array}{c}\text { No se } \\
\text { especifica }\end{array}$ \\
\hline [11] & $\begin{array}{l}\text { Programación } \\
\text { entera mixta }\end{array}$ & $\begin{array}{l}\text { Método de } \\
\text { Arcos } \\
\text { disyuntivos }\end{array}$ & $\begin{array}{l}\text { Nodos } \\
\text { adyacentes } \\
\text { con mayor } \\
\text { reducción en } \\
\text { la función } \\
\text { objetivo }\end{array}$ & $\begin{array}{l}\text { Asignación de } \\
\text { pesos a nodos } \\
\text { adyacentes }\end{array}$ & $\begin{array}{c}\text { No se } \\
\text { especifica }\end{array}$ & $\begin{array}{l}\text { Aspiración } \\
\text { por mejora } \\
\text { en función } \\
\text { objetivo }\end{array}$ \\
\hline [13] & $\begin{array}{l}\text { Límite Inferior } \\
\text { Lagrangeano }\end{array}$ & $\begin{array}{l}\text { Problemas } \\
\text { generados } \\
\text { aletoriamente }\end{array}$ & $\begin{array}{l}\text { Movimiento } \\
\text { No tabú con } \\
\text { mayor valor } \\
\text { en Función } \\
\text { Objetivo }\end{array}$ & $\begin{array}{c}\text { Matriz de } \\
\text { Frecuencia de } \\
\text { movimientos }\end{array}$ & $\begin{array}{l}\text { Matriz de } \\
\text { frecuencia de } \\
\text { movimientos }\end{array}$ & $\begin{array}{l}\text { Aspiración } \\
\text { por mejora } \\
\text { en función } \\
\text { objetivo }\end{array}$ \\
\hline
\end{tabular}

Tabla 2. Configuraciones básicas de la búsqueda tabú en modelos de lotificación multinivel. Fuente: Autores

\section{A. Solución de referencia}

Los métodos exactos de programación lineal y programación entera mixta son los más utilizados para comparar la eficiencia y la calidad (gap) de las soluciones arrojadas por los algoritmos de búsqueda tabú. Para problemas de gran tamaño, prevalece el uso de heurísticas y métodos de relajación.

\section{B. Solución Inicial}

Las soluciones utilizadas para inicializar la búsqueda tabú influyen en gran manera en el rendimiento de esta meta heurística. Según los artículos consultados, las heurísticas de solución Wagner-Within, lote por lote, transbordo y SPT son algunas de las alternativas utilizadas. Los métodos de generación de columnas, cadena de bloques y el método de arcos disyuntivos constituyen también una alternativa para su utilización en generación de soluciones para problemas de lotificación multinivel.

\section{Métodos de Intensificación}

En muchas de las soluciones al problema de lotificación de un solo nivel no se utilizaron (o no se especificaron) métodos de intensificación; esto debido principalmente al tamaño y complejidad de este tipo de problemas. El uso de memoria adaptativa es uno de los métodos de intensificación utilizados. En el caso de los modelos de lotificación multinivel, los criterios de intensificación se rigen por asignación de pesos o penalizaciones a los movimientos de la 
búsqueda.

\section{Métodos de Diversificación}

La memoria adaptativa es utilizada como método de diversificación. La definición de nuevas soluciones iniciales después de un número máximo de iteraciones sin mejora en la solución, es una estrategia de diversificación utilizada en la literatura. Adicionalmente, la creación de matrices de frecuencia para los movimientos realizados permite una mayor exploración del espacio de soluciones en problemas de lotificación multinivel.

\section{E. Método de Aspiración}

Para ambos tipos de problema, el método de aspiración más utilizado es la aspiración por objetivo, en la cual se extrae de la lista tabú el movimiento que represente una mejora en la función objetivo.

\section{F. Criterio de Parada}

Los criterios de parada más utilizados son el número máximo de iteraciones y el número máximo de iteraciones sin mejoras en la función objetivo.

\section{CONCLUSIONES}

La búsqueda tabú ha demostrado ser una alternativa eficiente para la solución de problemas de lotificación. La configuración de sus parámetros básicos depende principalmente del tipo y el tamaño del problema a resolver. De acuerdo con los contados aportes en la literatura científica que exponen de manera detallada el uso de la búsqueda tabú en la solución del problema de lotificación en sistemas multinivel, se puede evidenciar la necesidad del uso de métodos complejos de intensificación, diversificación y aspiración.

Se evidencia el gran potencial de la búsqueda tabú para la solución de problemas de lotificación de gran complejidad. Al tratarse de problemas de mayor tamaño y complejidad, la estructuración del problema mediante los métodos de arcos disyuntivos y cadena de bloques se convierten en unas de las principales alternativas para el abordaje del problema de lotificación multinivel.

La calidad de las soluciones iniciales para el algoritmo de búsqueda tabú influye en gran medida en su rendimiento computacional. Debido a esto, para problemas complejos, la selección del método de generación de soluciones iniciales debe buscar también un equilibrio entre la calidad y el tamaño del problema, buscando su simplificación o relajación.

\section{REFERENCIAS}

[1] F. Glover, "Tabu Search-Part I,” ORSA J. Comput., vol. 1, no. 3, pp. 190-206, Aug. 1989.

[2] F. Glover, “Tabu Search-Part II,” ORSA J. Comput., vol. 2, no. 1, pp. 4-32, Feb. 1990.

[3] F. Glover and E. Taillard, “A user's guide to tabu search,” Ann. Oper. Res., vol. 41, no. 1, pp. 1-28, Mar. 1993.

[4] K. S. Hindi, "Solving the single-item, capacitated dynamic lot-sizing problem with startup and reservation costs by tabu search," Comput. Ind. Eng., vol. 28, no. 4, pp. 701-707, 1995.

[5] K. S. Hindi, "Solving the CLSP by a Tabu Search Heuristic," J. Oper. Res. Soc., vol. 47, no. 1, pp. 151-161, 1996.

[6] M. Gopalakrishnan, K. Ding, J.-M. Bourjolly, and S. Mohan, “A Tabu-Search Heuristic for the Capacitated LotSizing Problem with Set-up Carryover,” Manage. Sci., vol. 47, no. 6, pp. 851-863, Jun. 2001.

[7] Y. F. Hung, C. P. Chen, C. C. Shih, and M. H. Hung, "Using tabu search with ranking candidate list to solve production planning problems with setups,” Comput. Ind. Eng., vol. 45, no. 4, pp. 615-634, 2003.

[8] B. Karimi, S. M. T. F. Ghomi, and J. M. Wilson, "A Tabu Search Heuristic for Solving the CLSP with Backlogging and Set-up Carry-over,” J. Oper. Res. Soc., vol. 57, no. 2, pp. 140-147, Feb. 2006.

[9] X. Li, F. Baki, P. Tian, and B. A. Chaouch, "A robust block-chain based tabu search algorithm for the dynamic lot sizing problem with product returns and remanufacturing," Omega, vol. 42, no. 1, pp. 75-87, Jan. 2014.

[10] R. Kuik, M. Salomon, L. N. Van Wassenhove, and J. Maes, "Linear Programming, Simulated Annealing and Tabu Search Heuristics for Lotsizing in Bottleneck Assembly Systems,” IIE Trans., vol. 25, no. 1, pp. 62-72, 
Jan. 1993.

[11] A. Kimms, "Competitive methods for multi-level lot sizing and scheduling: tabu search and randomized regrets," Int. J. Prod. Res., vol. 34, no. 8, pp. 2279-2298, Aug. 1996.

[12] Y.-F. Hung and K.-L. Chien, “A Multi-Class Multi-Level Capacitated Lot Sizing Model,” J. Oper. Res. Soc., vol. 51, no. 11, p. 1309, Nov. 2000.

[13] R. Berretta, P. M. França, and V. A. Armentano, "Metaheuristic approaches for the multilevel resourceconstrained lot-sizing problem with setup and lead times," Asia-Pacific J. Oper. Res., vol. 22, no. 02, pp. 261286, Jun. 2005.

[14] Ortiz Gaitán, S., \& Ruiz, C. (2017). Modelo de Programación Lineal Entera Mixta para la Programación de Sistemas tipo Job-Shop Flexible en Entornos Make to Order. INGE CUC, 13(2), 28-34. https://doi.org/10.17981/ingecuc.13.2.2017.03

[15] Oliva San Martín, C., \& Ramírez Guzmán, G. (2014). Algoritmo de tipo búsqueda tabú para un problema de programación de horarios universitarios vespertinos. INGE CUC, 9(2), 58-65. Recuperado a partir de https://revistascientificas.cuc.edu.co/ingecuc/article/view/7

[16] Sepúlveda Perico, A., Martínez Castro, R., Medina Roncancio, S., \& Salazar Arrieta, F. (2016). Proposed design of a network supply chain for the cocoa agro chain of Viota municipality, Cundinamarca. IJMSOR: International Journal of Management Science \& Operation Research, 1(1), 35-42. Retrieved from http://ijmsoridi.com/index.php/ijmsor/article/view/75 